\title{
Circular Polarization in Scintillating Sources
}

\author{
Jean-Pierre Macquart ${ }^{1}$, Lucyna Kedziora-Chudczer ${ }^{2}$, David \\ L. Jauncey ${ }^{2}$, R. Sault ${ }^{2}$, David P. Rayner ${ }^{2,3}$ \\ ${ }^{1}$ RCfTA, School of Physics, University of Sydney, NSW 2006, Australia \\ ${ }^{2}$ Australia Telescope National Facility, CSIRO, Australia \\ ${ }^{3}$ University of Tasmania, Hobart 7001, Tasmania, Australia
}

Abstract. We discuss the detection of variable circular polarization (CP) in several scintillating radio sources: PKS 1519-273, PKS 0405-385 and Sgr $A^{*}$. In all cases the $C P$ is variable, with the fractional variability in the $\mathrm{CP}$ greatly exceeding that in total intensity.

\section{Introduction}

Linear polarization observed in extragalactic radio sources is accepted as a characteristic signature of synchrotron emission. The characteristics and interpretation of the circular polarization (CP) observed in some extragalactic sources is unclear; the CP is typically weak and has been difficult to measure reliably. However, the design of the Australia Telescope Compact Array (ATCA) makes it capable of high-accuracy circular polarimetry. We present measurements of circular polarization in three variable compact radio sources.

\section{Results}

PKS 1519-273 is a $z \gtrsim 0.4, m_{V}=18.5$ BL Lac object. The source exhibits persistent intraday variability: it has been observed to vary during each of the 7 times it has been observed with the ATCA. We interpret the source's variability in terms of interstellar scintillation. A lower limit on the distance to the scattering screen (from its angular size at $1.7 \mathrm{GHz}$ ) limits the angular size to $\theta_{S}=15-35 \mu$ as and the brightness temperature of the source to $T_{B} \gtrsim 5 \times 10^{13} \mathrm{~K}$ at $4.8 \mathrm{GHz}$. The strong correlation between Stokes $I$ and $V$ suggests a common origin within the source, and enables us to determine the degree of circular polarization of the polarized sub-component within the source. The degree of circular polarization is extremely high for an extragalactic source: $-3.4 \pm 0.4 \%$ at $4.8 \mathrm{GHz}$. We detect no changes in the sign of the CP as a function of frequency between 1.4 and $8.6 \mathrm{GHz}$. See Macquart et al. (2000) for further details.

PKS 0405-385 is a $m_{V}=18.5, z=1.285$ quasar which exhibited largeamplitude variability on a 40 min timescale at centimetre wavelengths for the two months commencing June 1996. The variability is interpreted in terms of scintillation (Kedziora-Chudczer et al. 1997). Another outburst of rapid variability was observed from November 1998 to April 1999. Figure 1 shows the polarization variability of PKS 0405-385 for Jan 15 1999. The mean CP over this period was $\langle V\rangle /\langle I\rangle \approx 0.2 \%$. In both the 8 and $5 \mathrm{GHz}$ data presented here, the $\mathrm{CP}$ is strongly correlated with the intraday variability (IDV) in total flux density, and is delayed by approximately 0.05 days. This suggests that the circularly polarized region in the source occupies a similar sized volume but is slightly displaced from the component that shows the strong IDV in total flux density. However, the interpretation of the CP is not always simple, since the 
correlation between $V$ and $I$ changed character over the course of the outburst, showing both positive, negative and at times no correlation.

Sgr $\mathrm{A}^{*}$ has been observed to exhibit variable CP at 1.4 and $2.5 \mathrm{GHz}$. The CP variability timescale is $\lesssim 20$ days, with its fractional variability exceeding that in the total intensity. If the CP is intrinsic to the source (as opposed to propagationinduced), this implies that the CP comes from only a sub-component of the source, and that the degree of $\mathrm{CP}$ of this sub-source is $V_{s} / I_{s}<-2.3 \%$ at $1.4 \mathrm{GHz}$ and $V_{s} / I_{s}=-2.1_{-1.7}^{+0.8} \%$ at $2.5 \mathrm{GHz}$. The mean degree of circular polarization is $\langle V\rangle /\langle I\rangle \approx-0.3 \%$.

\section{Origin of the Circular Polarization}

In all three objects the origin of the CP is unclear. However, for the IDV sources it is clearly associated with scintillation, and it is possible that it may be caused by the scintillation process (Macquart \& Melrose 2000a,b). The characteristics of the CP observed in PKS 1519-273 belie the attribution of the CP to any known mechanism, including the small amount associated with synchrotron emission.

Acknowledgments. The Australia Telescope is funded by the Commonwealth Government for operation as a national facility by the CSIRO.

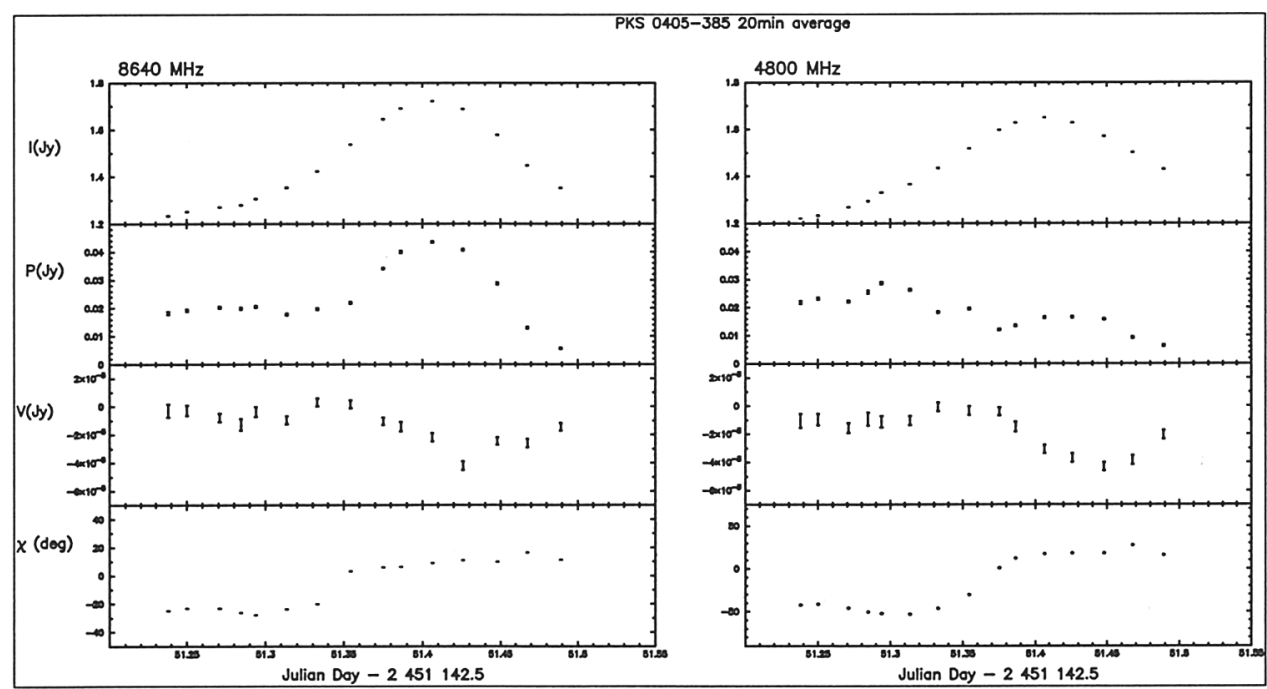

Figure 1. Variability of PKS 0405-385 in the total intensity $(I), \mathrm{CP}$ $(V)$, and magnitude and position angle of the linear polarization $(P$ and $\chi$ ) at 4.80 and $8.64 \mathrm{GHz}$ over 6 hours on 15 Jan 99. Each point represents a 20 min average, and is plotted with $1 \sigma$ error bars.

\section{References}

Kedziora-Chudczer, L., Jauncey, D.L., Wieringa, M.H., Walker, M.A., Nicolson, G.D., Reynolds, J.E. \& Tzioumis, A.K., 1997, Ap.J., 490, L9

Macquart, J.-P., Kedziora-Chudczer, L., Jauncey, D.L., Rayner, D.P., 2000, Ap.J., 538, 623

Macquart, J.-P., Melrose, D.B., 2000a, Phys.Rev.E, 62, 4177

Macquart, J.-P., Melrose, D.B., 2000b, to appear in Ap.J., 545 